\title{
Consenso experto sobre el uso clínico de los tratamientos por vía tópica en el manejo del dolor neuropático periférico
}

\author{
C. Pérez ${ }^{1}$, M. J. Rodríguez ${ }^{2}$, A. Guerrero ${ }^{3}$, C. Margarit ${ }^{4}$, C. Martín-Estefanía ${ }^{5}$, A. Oteo-Álvaro ${ }^{6}$ y F. Caballero ${ }^{7}$ \\ ${ }^{1}$ Hospital Universitario La Princesa. Madrid. ${ }^{2}$ Hospital Regional Universitario Carlos Haya. Málaga. \\ ${ }^{3}$ Hospital Clínico San Carlos. Madrid. ${ }^{4}$ Hospital General Universitario. Baza, Alicante. ${ }^{5}$ Hospital Clínico \\ Universitario San Juan de Alicante. Alicante. ${ }^{6}$ Hospital Universitario Gregorio Marañón. Madrid. \\ ${ }^{7}$ Academia de Medicina. Facultad de Ciencias Biosanitarias. Universidad Francisco de Vitoria. Pozuelo de \\ Alarcón, Madrid
}

Pérez C, Rodríguez MJ, Guerrero A, Margarit C, Martin-Estefanía C, Oteo-Älvaro A, Caballero F. Consenso experto sobre el uso clínico de los tratamientos por vía tópica en el manejo del dolor neuropático periférico. Rev Soc Esp Dolor 2013; 20(6): 308-323.

\begin{abstract}
Objective: To propose consensus from a panel of state level that integrates clinical experience and the most current evidence, recommendations on the clinical use of topical treatments for the management of peripheral neuropathic pain (PNP).

Methods: We propose, based on a literature review on topical therapeutic options in PNP, a series of professional standards and clinical recommendations for improving the use of these topical agents. We used the modified Delphi method in two rounds to contrast the views of a national panel of 52 renowned experts, selected by a "snowball" strategy among the group of anesthesiologists pain units (94\%) and other specialists (neurologist and trauma). We evaluated 61 clinical recommendations grouped into 6 areas: a) PNP systemic versus topical treatment (11 items); b) postsurgical neuropathic pain, post-traumatic and painful stumps (12 items); c) post-herpetic neuralgia, intercostal and trigeminal (9 items); d) PNP entrapment (8 items); e) CRPS (11 items); and f) diabetic neuropathy (DN) and other polyneuropathy (HIV, alcohol, toxicity, etc.) (10 items). We used a Likert-type ordinal scale of 9 points (disagree/agree) to evaluate each recommendation. After the first round of the survey, information was provided requested to reconsider the vote on items not agree.
\end{abstract}

Financiación: El laboratorio Astellas ha dado soporte al comité científico para financiar el desarrollo de las tareas de campo de la encuesta Delphi del estudio, sin participar en las tareas de diseño, recogida de información, análisis de datos, ni en la redacción del presente artículo.

Recibido: 30-05-13.

Aceptado: 01-07-13
Results: After the first round the panel consensus was achieved in 37 of the 61 issues raised. At the end of the second round of the agreement amounted to 46 (75\%). In general, there was consensus among experts on whether to introduce topical treatment in first line treatment of PNP and its greater acceptance by patients compared with systemic. He was also a shared view consider in the management of various types of PNP. Also reached a high level of agreement to accept, from a physiological point of view, the indication for treatment with capsaicin patch $8 \%$ for various types of PNP.

Conclusions: Experts in the clinical management of PNP show a high level of professional agreement with various therapeutic recommendations for study. The dissemination of such recommendations can help improving the routine management of topical drugs for neuropathic pain in our health system.

Key words: Topical therapy. Consensus. Peripheral neuropathic pain.

\section{RESUMEN}

Objetivo: Proponer a partir del consenso de un panel de expertos de ámbito estatal que integre la experiencia clínica y la evidencia disponible más actual, recomendaciones sobre el uso clínico de los tratamientos por vía tópica para el manejo del dolor neuropático periférico (DNP).

Métodos: Se proponen, a partir de una revisión bibliográfica sobre las distintas opciones terapéuticas tópicas en DNP, una serie de criterios profesionales y recomendaciones clínicas para la mejora del uso de dichos agentes tópicos. Se empleó el método Delphi modificado en dos rondas para contrastar las opiniones de un panel nacional de 52 reconocidos expertos, seleccionados mediante una estrategia en "bola de nieve" de entre el colectivo de anestesiólogos de unidades del dolor (94 \%) y otros especialistas (neurólogos y traumatólogos). Se evaluaron 61 recomendaciones clínicas agrupadas en 6 áreas temáticas: a) DNP: tratamiento tópico versus sistémico (11 ítems); b) dolor neuropático postquirúrgico, postraumático y muñones dolorosos (12 ítems); 
c) neuralgia posherpética, intercostal y del trigémino (9 ítems); d) DNP por atrapamiento (8 ítems); e) síndrome de dolor regional complejo (11 ítems); y f) neuropatía diabética (ND) y otras polineuropatías (por VIH, alcohol, toxicidad, etc.) (10 ítems). Se empleó una escala ordinal de tipo Likert de 9 puntos (desacuerdo/acuerdo) para evaluar cada recomendación. Tras la primera ronda de encuesta, se facilitó al panel información del resultado (resultados estadísticos y opiniones libres de los panelistas) y se solicitó la reconsideración del voto sobre los ítems no consensuados.

Resultados: Tras la primera ronda del panel se logró consenso en 37 de las 61 cuestiones planteadas. Al final de la segunda ronda el acuerdo ascendió hasta 46 ítems (75 \%). En general, se aprecia consenso entre los expertos sobre la conveniencia de introducir los tratamientos tópicos en primera línea de tratamiento del DNP y sobre su mejor aceptación por los pacientes frente a los sistémicos. Asimismo, fue criterio compartido que la combinación de estos fármacos tópicos con los tratamientos sistémicos es una opción a considerar en el manejo de varios tipos de DNP. También se alcanzó un alto grado de acuerdo en aceptar, desde un punto de vista fisiopatológico, la indicación del tratamiento con parche de capsaicina al $8 \%$ para varios tipos de DNP.

Conclusión: Los expertos en el manejo clínico del DNP muestran un elevado nivel de acuerdo profesional con diversas recomendaciones terapéuticas analizadas en el estudio. La difusión de tales recomendaciones puede ayudar a la mejora del manejo rutinario de fármacos tópicos para el dolor neuropático en nuestro sistema sanitario.

Palabras clave: Terapia tópica. Consenso. Dolor neuropático periférico.

\section{INTRODUCCIÓN}

La Sociedad Internacional para el Estudio del Dolor (IASP, International Association for the Study of Pain) define el dolor neuropático (DN) como un dolor iniciado o causado por una lesión o disfunción primaria del sistema nervioso, ya sea periférico o central. Es el dolor que aparece como consecuencia de lesiones del propio sistema de recepción, transmisión y análisis de los estímulos nociceptivos, y no por la captación de estímulos originados fuera del sistema nervioso, pudiendo por tanto tener su origen en cualquier punto de las vías de conducción nerviosa. Para evitar problemas con el término disfunción, un grupo europeo experto en el estudio del dolor propuso una nueva definición: es aquel dolor que es consecuencia directa de una lesión o enfermedad que afecta al sistema somatosensorial (1).

Aunque la prevalencia del DN no ha sido estudiada con suficiente rigor, se estima que puede afectar al $3 \%$ de la población a nivel mundial. Gálvez y cols. revisaron la bibliografía española entre 1990 y 2004 con objeto de determinar su incidencia nacional (2). Este estudio no consiguió generalizar unas conclusiones fiables por la variabilidad de criterios de diagnóstico empleados y por la ausencia de un registro fiable de pacientes. Se desconoce, pues, su epidemiología real en nuestro país, aunque existen dos estudios recientes sobre la cuestión: uno de ellos (3), realizado en atención primaria, sugiere que la incidencia puede llegar al $25 \%$ de los pacientes que consultan por dolor crónico (incluyendo el dolor mixto); el otro, sobre pacientes atendidos en unidades del dolor (4), identifica al DN como el motivo de consulta en el 51,9\% de los sujetos atendidos. A nivel nacional, se estima que el DN puede afectar a más de dos millones de personas.

El DN puede influir de forma significativa sobre el funcionamiento cotidiano y la calidad de vida de los pacientes, tanto en sus aspectos físicos como psicológicos. Muchas personas que sufren el trastorno tienen dificultades para dormir, refieren problemas para concentrarse, falta de energía y somnolencia, y ven limitada su capacidad para trabajar o para abordar con autonomía tareas comunes, como vestirse o caminar. Además, estos trastornos suelen conllevar un coste económico importante, ya que los pacientes pueden necesitar diferentes métodos para aliviar el dolor y utilizar muchos tratamientos, sin llegar, en ocasiones, a encontrar uno definitivo.

El DN periférico es un grupo complejo de síndromes dolorosos, en el que se involucran distintas causas y mecanismos patogénicos, y cuyo abordaje terapéutico continúa siendo un reto clínico difícil. Se dispone para ello de una amplia batería de tratamientos farmacológicos (analgésicos, antidepresivos, antiepilépticos, opiáceos, agentes tópicos, etc.) y no farmacológicos (neuromodulación, radiofrecuencia, bloqueos nerviosos, técnicas neuroquirúrgicas, etc.) $(5,6)$. Pese a ello, entre el $40-60 \%$ de los pacientes afectados consiguen un alivio insuficiente de sus molestias o perciben efectos adversos molestos de los analgésicos ensayados. Su complejidad fisiopatológica, las distintas formas clínicas de presentación que adopta y la falta de pruebas diagnósticas específicas hacen muy común el manejo inadecuado de estos pacientes, ya de por sí dificultoso por la eficacia parcial de los tratamientos disponibles. Por todo ello, el DN resulta un problema frecuentemente infradiagnosticado e infratratado. Afortunadamente, en los últimos años se ha alcanzado una mejor compresión de los mecanismos subyacentes de estos síndromes dolorosos, lo que está permitiendo desarrollar estrategias de tratamiento más efectivas (7).

En España existe una gran variabilidad de prácticas en el manejo del DN, en parte, debida a que los pacientes son atendidos por diversos especialistas de distintas áreas médicas. No obstante, existen múltiples guías clínicas y documentos de revisión que orientan la actitud diagnóstica y terapéutica sobre la base de la evidencia disponible (8-12). El último consenso publicado en España, con participación de siete sociedades científicas, resume la literatura disponible 
hasta 2009 (13). En 2010 se han comercializado en España tres fármacos para el $\mathrm{DN}$ periférico, dos de ellos tratamientos tópicos y el otro sistémico, que no están aún incluidos en las guías terapéuticas españolas ni europeas (14).

Con el presente estudio se ha pretendido recabar el criterio experto de especialistas en dolor para valorar el posicionamiento clínico de los nuevos tratamientos tópicos en el manejo del DN periférico, a partir de la bibliografía científica disponible y de la experiencia clínica personal de los panelistas que participan en este proyecto. Se pretende, con ello, desarrollar unas recomendaciones expertas sobre el uso clínico de dichos agentes, incidiendo particularmente en cuestiones aún sometidas a controversia profesional.

En el desarrollo del estudio se ha empleado el método Delphi modificado (15), una técnica estructurada de consenso profesional a distancia, variante del procedimiento original desarrollado por Dalkey y cols. en Rand Corporation Santa Mónica (California) (16,17), que mantiene sus principales ventajas frente a otras alternativas técnicas (como las conferencias de consenso, los grupos nominales o las reuniones no estructuradas) y resuelve algunos de sus principales inconvenientes (18).

\section{MATERIAL Y MÉTODOS}

El método Delphi modificado solicita la opinión individual y anónima de cada experto sobre el tema a debate mediante la respuesta en dos rondas a una encuesta estructurada escrita, vehiculada por correo. La repetición de las rondas de encuesta permite la reconsideración y aproximación de posturas divergentes. El presente estudio se desarrolló en cuatro fases: 1) constitución de un comité científico, responsable de la selección y formulación de los ítems de encuesta (criterios profesionales y recomendaciones clínicas sometidas a debate); 2) selección de un panel de expertos, con especial interés e implicación en el tratamiento del DN; 3) encuesta postal en dos rondas con procesamiento intermedio y feedback de resultados a los panelistas; y 4) análisis de resultados y discusión de conclusiones en sesión presencial del comité científico.

El comité científico impulsor del proyecto, junto a un asesor metodológico externo, seleccionó y redactó los contenidos del cuestionario Delphi tras un proceso de revisión bibliográfica sistematizada. Cada ítem del cuestionario es una propuesta (afirmativa o negativa) de criterio profesional o recomendación clínica sobre terapia tópica del DN, en cualquier aspecto de interés o controversia. La versión final del cuestionario incluyó 61 ítems (Tabla I) agrupados en 6 áreas temáticas: a) DN periférico: tratamiento tópico versus sistémico (11 ítems); b) DN posquirúrgico, postraumático y muñones dolorosos (12 ítems); c) neuralgia posherpética, intercostal y del trigémino (9 ítems); d) DN periférico por atrapamiento (8 ítems); e) síndrome de dolor regional complejo (11 ítems); y f) neuropatía diabética y otras polineuropatías (por VIH, alcohol, toxicidad, etc.) (10 ítems).

TABLA I. RESUMEN DE RESULTADOS ESTADÍSTICOS DEL CONSENSO EXPERTO SOBRE EL USO CLÍNICO DE LOS TRATAMIENTOS POR VÍA TÓPICA EN EL MANEJO DEL DOLOR NEUROPÁTICO PERIFÉRICO Y NIVEL DE CONSENSO GRUPAL ALCANZADO (VER LEYENDA A PIE DE TABLA)

\section{Dolor neuropático periférico (DNP): tratamiento tópico versus sistémico}

\begin{tabular}{|c|c|c|c|c|}
\hline & Mediana & $\begin{array}{l}\% \text { panelistas } \\
\text { a favor }\end{array}$ & Promedio & $\begin{array}{c}\text { Rango } \\
\text { intercuartílico }\end{array}$ \\
\hline $\begin{array}{l}\text { 1. La vía tópica y la sistémica han demostrado eficacias } \\
\text { similares en cuanto al porcentaje de pacientes } \\
\text { respondedores }\end{array}$ & $7(*)$ & 50,0 & 5,88 & 2 \\
\hline $\begin{array}{l}\text { 2. Los tratamientos tópicos obtienen mejor respuesta en } \\
\text { la mejoría de los síntomas y signos positivos frente } \\
\text { a los fármacos sistémicos (por ejemplo, alodinia, } \\
\text { disestesias, crisis lancinantes y dolor urente) }\end{array}$ & $5(*)$ & 45,5 & 5,73 & 2 \\
\hline $\begin{array}{l}\text { 3. Los tratamientos tópicos frente a los sistémicos } \\
\text { mejoran otros parámetros como la calidad de vida } \\
\text { debido a la ausencia de efectos adversos }\end{array}$ & 7 & 84,0 & 7,28 & 1 \\
\hline $\begin{array}{l}\text { 4. Los tratamientos tópicos no precisan de profilaxis de } \\
\text { efectos secundarios }\end{array}$ & $7(*)$ & 64,4 & 6,53 & 4 \\
\hline $\begin{array}{l}\text { 5. Todos los pacientes con DNP son candidatos a } \\
\text { tratamiento tópico }\end{array}$ & $5(*)$ & 11,7 & 4,71 & 5 \\
\hline
\end{tabular}


TABLA I (CONT.). RESUMEN DE RESULTADOS ESTADÍSTICOS DEL CONSENSO EXPERTO SOBRE EL USO CLÍNICO DE LOS TRATAMIENTOS POR VÍA TÓPICA EN EL MANEJO DEL DOLOR NEUROPÁTICO PERIFÉRICO Y NIVEL DE CONSENSO GRUPAL ALCANZADO (VER LEYENDA A PIE DE TABLA)

1. Dolor neuropático periférico (DNP): tratamiento tópico versus sistémico

\begin{tabular}{|c|c|c|c|c|}
\hline & Mediana & $\begin{array}{l}\% \text { panelistas } \\
\text { a favor }\end{array}$ & Promedio & $\begin{array}{l}\text { Rango } \\
\text { intercuartílico }\end{array}$ \\
\hline $\begin{array}{l}\text { 6. El número de abandonos es mayor en los } \\
\text { tratamientos sistémicos que en los tópicos }\end{array}$ & 8 & 78,0 & 7,22 & 2 \\
\hline $\begin{array}{l}\text { 7. Se pueden combinar tratamientos tópicos y } \\
\text { tratamientos sistémicos en el DNP }\end{array}$ & 9 & 100,0 & 8,58 & 1 \\
\hline $\begin{array}{l}\text { 8. Es recomendable introducir la vía tópica en primera } \\
\text { línea del tratamiento del DNP }\end{array}$ & 7 & 72,2 & 6,96 & 2 \\
\hline $\begin{array}{l}\text { 9. Los tratamientos tópicos son mejor aceptados por los } \\
\text { pacientes frente a los sistémicos }\end{array}$ & 8 & 84,0 & 7,62 & 2 \\
\hline $\begin{array}{l}\text { 10. La eficacia de los tratamientos tópicos que necesitan } \\
\text { una o varias aplicaciones diarias (por ejemplo, } \\
\text { parche de lidocaína, capsaicina en crema) está } \\
\text { limitada al tiempo de aplicación }\end{array}$ & $7(*)$ & 66,7 & 6,25 & 1 \\
\hline $\begin{array}{l}\text { 11. Los fármacos con dianas farmacológicas periféricas } \\
\text { deberían ser los indicados en el tratamiento tópico } \\
\text { del DNP }\end{array}$ & 7 & 77,1 & 7,19 & 1 \\
\hline
\end{tabular}

\section{Dolor neuropático posquirúrgico, postraumático y muñones dolorosos}

12. El uso del parche de capsaicina al $8 \%$ en fases tempranas en pacientes que han sufrido este tipo de lesiones puede evitar la sensibilización a nivel

8 central que suele producirse a largo plazo

13. Un diagnóstico incorrecto del DNP conlleva a menudo fracaso terapéutico

14. La afectación en este tipo de lesiones del SNP justifica un tratamiento tópico que actúe a nivel de SNP

15. El tratamiento correcto del DNP es uno de los más difíciles de conseguir

16. El reducido número de aplicaciones del tratamiento tópico en este tipo de pacientes facilita el cumplimiento terapéutico

17. Para un correcto tratamiento del dolor neuropático, la escala de valoración DN4 puede ser de gran utilidad

18. Los fármacos que en sus ensayos clínicos hayan demostrado su eficacia en estos tres modelos de dolor neuropático (neuralgia posherpética, VIH y neuropatía diabética) serán eficaces en otros tipos de DNP

19. La duración de los distintos ensayos clínicos aleatorizados nunca ha sido superior a los tres meses, pero sus resultados son válidos en la práctica clínica diaria
8

8

8

8

8

7
7,14

2

1

1

1

1

2

$\begin{array}{lll}68,7 & 7,02 & 2\end{array}$

$\begin{array}{lll}74,5 & 6,55 & 1\end{array}$ 
TABLA I (CONT.). RESUMEN DE RESULTADOS ESTADÍSTICOS DEL CONSENSO EXPERTO SOBRE EL USO CLÍNICO DE LOS TRATAMIENTOS POR VÍA TÓPICA EN EL MANEJO DEL DOLOR NEUROPÁTICO PERIFÉRICO Y NIVEL DE CONSENSO GRUPAL ALCANZADO (VER LEYENDA A PIE DE TABLA)

2. Dolor neuropático posquirúrgico, postraumático y muñones dolorosos

Mediana $\begin{gathered}\% \text { panelistas } \\ \text { afavor }\end{gathered} \quad$ Promedio $\begin{gathered}\text { Rango } \\ \text { intercuartílico }\end{gathered}$

20. Los antidepresivos (tricíclicos y duales) y los ligandos alfa-2-delta de los canales de calcio se consideran como tratamiento de primera línea del 8 81,8 7,96 DNP en diversas guías clínicas

21. El uso del parche de capsaicina al $8 \%$ en este tipo de pacientes ha demostrado ser eficaz y permite la reducción del uso de medicación oral y concomitante

22. La combinación del parche de capsaicina al $8 \%$ con otros fármacos orales de primera línea es una alternativa eficaz para el tratamiento del DNP

23. El parche de capsaicina al $8 \%$ se puede utilizar como primera opción de tratamiento en dolor neuropático postquirúrgico, postraumático y muñones dolorosos

3. Neuralgia posherpética (NPH), intercostal y del trigémino** (pág. 323)

24. El parche de capsaicina al $8 \%$ puede ser un fármaco de primera línea en el tratamiento de la NPH

25. En el caso de la neuralgia del trigémino que afecte al territorio V1 (ya que no se recomienda su aplicación en la cara), el parche de capsaicina al $8 \%$ es una alternativa de tratamiento ante una respuesta insuficiente con otros fármacos por vía oral (antiepilépticos, antidepresivos)

26. En un paciente con neuralgia intercostal localizada, el parche de capsaicina al $8 \%$ se considera una opción válida para su tratamiento

27. Desde un punto de vista fisiopatológico se entiende la indicación del tratamiento con parche de capsaicina al $8 \%$ en todo tipo de DNP

28. En el territorio trigeminal no se considera una opción de tratamiento el parche de capsaicina al $8 \%$, por no estar indicada su utilización en la cara y el cuero cabelludo

29. En caso de respuesta parcial con la aplicación del primer parche de capsaicina al $8 \%$ en alguno de estos casos (NPH, intercostal y trigémino), intentaría una segunda aplicación pasados dos meses

30. Se puede utilizar el parche de capsaicina al $8 \%$ como primera opción de tratamiento en la neuralgia intercostal
7

80,0

7,40

1

8

86

7,64

7

75,5

6,63

39,2

5,04

4 
TABLA I (CONT.). RESUMEN DE RESULTADOS ESTADÍSTICOS DEL CONSENSO EXPERTO SOBRE EL USO CLÍNICO DE LOS TRATAMIENTOS POR VÍA TÓPICA EN EL MANEJO DEL DOLOR NEUROPÁTICO PERIFÉRICO Y NIVEL DE CONSENSO GRUPAL ALCANZADO (VER LEYENDA A PIE DE TABLA)

3. Neuralgia posherpética (NPH), intercostal y del trigémino** (pág. 323)

Mediana $\begin{gathered}\% \text { panelistas } \\ \text { afavor }\end{gathered} \quad$ Promedio $\begin{gathered}\text { Rango } \\ \text { intercuartílico }\end{gathered}$

31. El tratamiento con parches de capsaicina al $8 \%$ en estas indicaciones (NPH, intercostal y trigémino) puede tener más ventajas que inconvenientes

$\begin{array}{llll}7 & 80,00 & 7,38 & 2\end{array}$

32. La evidencia existente para tratar con el parche de capsaicina al $8 \%$ la neuralgia intercostal y trigeminal es suficiente

$6(*) \quad 21,3 \quad 5,32 \quad 4$

4. Dolor neuropático periférico por atrapamiento

33. La titulación necesaria en los tratamientos sistémicos en DNP por atrapamiento no es necesaria en el caso de los tratamientos tópicos

34. En el síndrome de túnel carpiano (STC) leve o moderado sin signos de denervación en la electromiografía estaría indicado el tratamiento conservador y, entre ellos, la vía tópica

35. En el tratamiento del STC el parche de capsaicina al $8 \%$ permite aliviar los síntomas localizados sin los efectos secundarios de los tratamientos orales

7

36. Los tratamientos tópicos frente a los sistémicos tienen menos efectos adversos sistémicos, no hay que titular la dosis y no tienen interacciones farmacológicas

37. Cuanto antes se utilice el parche de capsaicina al $8 \%$, menos sensibilización central y resistencias terapéuticas tendrá el paciente y la posibilidad de respuesta terapéutica será mayor

38. La utilización del parche de capsaicina al $8 \%$ es una alternativa terapéutica en el tratamiento de las neuropatías por atrapamiento

39. Tanto la cirugía abierta tradicional como las técnicas de mínima incisión y las técnicas endoscópicas se

40. En el neuroma de Morton de pequeño tamaño el parche de capsaicina al $8 \%$ podría ser una primera opción antes de plantear un tratamiento quirúrgico

\section{Síndrome de dolor regional complejo (SDRC)}

41. El inicio temprano del tratamiento en el SDRC reduce las posibilidades de su cronificación

42. En la fase aguda del SDRC es aconsejable la inmovilización de la extremidad/extremidades afectas
9

8

80,9

7,04

1

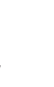


TABLA I (CONT.). RESUMEN DE RESULTADOS ESTADÍSTICOS DEL CONSENSO EXPERTO SOBRE EL USO CLÍNICO DE LOS TRATAMIENTOS POR VÍA TÓPICA EN EL MANEJO DEL DOLOR NEUROPÁTICO PERIFÉRICO Y NIVEL DE CONSENSO GRUPAL ALCANZADO (VER LEYENDA A PIE DE TABLA)

5. Síndrome de dolor regional complejo (SDRC)

\begin{tabular}{|c|c|}
\hline Mediana & $\begin{array}{c}\% \text { panelistas } \\
\text { a favor }\end{array}$ \\
\hline
\end{tabular}

43. El uso de opioides por vía oral en el SDRC contraindica el tratamiento concomitante con parches de opioides transdérmicos

44. En el control del SDRC el tratamiento tópico sería el de elección

45. En la fase "caliente" del SDRC está contraindicado cualquier tipo de tratamiento tópico

2

46. Los antiinflamatorios no esteroideos (AINE) aplicados de forma tópica consiguen un adecuado control del dolor en el SDRC

47. Los opioides en formulación transdérmica constituyen el grupo farmacológico de primera elección en el tratamiento del SDRC

48. El tratamiento tópico con dimetilsulfóxido al $50 \%$ ha demostrado eficacia en el control del dolor del SDRC

$$
76,6
$$

49. La aplicación de parches de capsaicina tópica al $8 \%$ es una opción terapéutica válida en el tratamiento del dolor en el SDRC

$\begin{array}{llll}7(*) & 64,6 & 6,17 & 2\end{array}$

50. La lidocaína tópica no consigue aliviar el dolor en la zona de aplicación en los casos de SDRC

$5(*) \quad 59,6 \quad 4,49 \quad 2$

51. La utilización de parches de capsaicina al $8 \%$ en el SDRC se planteará cuando hayan fracasado otros tratamientos tópicos

$\begin{array}{llll}4(*) & 18,7 & 4,58 & 4\end{array}$

6. Neuropatía diabética (ND)*** (pág. 323) y otras polineuropatías (por VIH, alcohol, toxicidad, etc.)

52. En este tipo de pacientes el tratamiento tópico reduciría el riesgo de interacciones medicamentosas

53. Una evaluación clínica precisa de la situación neurológica del paciente puede contribuir al éxito terapéutico

54. En el paciente con polineuropatía diabética se debería aplicar un tratamiento individualizado

55. En la medicación para el dolor neuropático las interacciones posibles son un aspecto poco relevante

56. El tratamiento tópico, al no presentar interacciones medicamentosas, debería ser el tratamiento de primera elección

57. Los fármacos tópicos son tratamientos de primera línea de las polineuropatías

$\begin{array}{cccc}8 & 88,0 & 7,56 & 2 \\ 9 & 92,0 & 8,18 & 1 \\ 8 & 94,0 & 8,06 & 2 \\ 2 & 86,0 & 2,54 & 2 \\ 7(*) & 58,3 & 6,38 & 1 \\ 6(*) & 23,3 & 5,49 & 4\end{array}$


TABLA I (CONT.). RESUMEN DE RESULTADOS ESTADÍSTICOS DEL CONSENSO EXPERTO SOBRE EL USO CLÍNICO DE LOS TRATAMIENTOS POR VÍA TÓPICA EN EL MANEJO DEL DOLOR NEUROPÁTICO PERIFÉRICO Y NIVEL DE CONSENSO GRUPAL ALCANZADO (VER LEYENDA A PIE DE TABLA)

6. Neuropatía diabética (ND)*** (pág. 323) y otras polineuropatías (por VIH, alcohol, toxicidad, etc.)

\begin{tabular}{|c|c|c|c|c|}
\hline & Mediana & $\begin{array}{l}\% \text { panelistas } \\
\text { a favor }\end{array}$ & Promedio & $\begin{array}{l}\text { Rango } \\
\text { intercuartílico }\end{array}$ \\
\hline $\begin{array}{l}\text { 58. El parche de capsaicina al } 8 \% \text { es el único que ha } \\
\text { demostrado eficacia en la neuropatía por VIH }\end{array}$ & 7 & 72,9 & 6,69 & 1 \\
\hline $\begin{array}{l}\text { 59. El bloqueo de los canales TRPV1 en este tipo de } \\
\text { pacientes es la clave para el éxito del tratamiento de } \\
\text { las polineuropatías }\end{array}$ & 7 & 75,0 & 6,85 & 1 \\
\hline $\begin{array}{l}\text { 60. El tratamiento tópico permite reducir efectos } \\
\text { adversos en este tipo de pacientes }\end{array}$ & 8 & 90,0 & 7,78 & 2 \\
\hline $\begin{array}{l}\text { 61. En este tipo de pacientes el abordaje temprano con } \\
\text { tratamientos tópicos podría ser la primera opción } \\
\text { terapéutica }\end{array}$ & 7 & 79,2 & 6,81 & 0 \\
\hline
\end{tabular}

$\%$ panelistas a favor = porcentaje de panelistas que puntúan dentro de la región de tres puntos que contiene la mediana ([1-3], [4-6], [7-9]). Según los criterios de interpretación descritos en la metodología, todos los items resultan aceptados por consenso grupal, excepto los señalados con (*): items 1, 2, 4, 5, 10, 25, 28, 32, 45, 48, 49, 50, 51, 56, 57.

La encuesta empleó una escala ordinal tipo Likert de nueve puntos ( 1 = pleno desacuerdo; 9 = pleno acuerdo), adaptada de las empleadas en los procedimientos de evaluación de tecnologías sanitarias (19), con las categorías de respuesta agrupadas mediante cualificadores lingüísticos en tres regiones (1-3 = "desacuerdo"; 4-6 = "ni acuerdo, ni en desacuerdo"; 7-9 = "acuerdo"). El cuestionario recogió también observaciones libres a los panelistas.

Los expertos seleccionados por el comité científico para formar parte del panel fueron profesionales con especial interés y reconocido criterio en DN. Para su identificación se empleó una estrategia en "bola de nieve" (20) a partir de los contactos personales de los miembros del comité. Tras este proceso, aceptaron participar 52 profesionales, 49 anestesiólogos de unidades de dolor, 2 neurólogos y 1 traumatólogo (Anexo 1). El trabajo de campo del proyecto se desarrolló durante cuatro semanas, entre febrero y marzo del año 2012, por vía electrónica.

Para analizar la opinión grupal y el tipo de consenso alcanzado sobre cada cuestión planteada, se emplea la posición de la mediana de puntuaciones del grupo y el "nivel de concordancia" alcanzado por los encuestados (21). Se considera consensuado un ítem cuando existe "concordancia" de opinión en el panel, esto es, cuando los expertos que puntúan fuera de la región de tres puntos ([1-3], [4-6], [7-9]) que contiene la mediana son menos de la tercera parte de los encuestados. En tal caso, el valor de la mediana determina el consenso alcanzado: "desacuerdo" grupal con el ítem, si la mediana es $\leq 3$, o "acuerdo" grupal con el ítem si la mediana es $\geq 7$. Los casos en los que la mediana se encuentra en la región 4-6 son considerados ítems "dudosos" para el grupo. Por el contrario, se establece que existe "discordancia" de criterio en el panel cuando las puntuaciones de un tercio o más de los panelistas están en la región [1-3], y de otro tercio o más en la región [7-9]. Los ítems restantes en los que no se observa concordancia ni discordancia se consideran con un nivel de consenso "indeterminado". Todos los ítems en los que el grupo no alcanzó un consenso a favor o en contra de la cuestión planteada se propusieron a la reconsideración del panel en la segunda ronda Delphi, informando a los panelistas de la distribución detallada de las respuestas y comentarios del grupo en la primera encuesta. También se sometieron a revaluación los ítems en los que se apreció una alta dispersión de opiniones (rango intercuartílico $\geq 4$ puntos).

\section{RESULTADOS}

Los 52 expertos consultados completaron las dos rondas de evaluación y no propusieron nuevos ítems. En la primera ronda se consensuaron 37 de las 61 cuestiones analizadas según los criterios preestablecidos (32 de ellos por acuerdo grupal y 5 de ellos por desacuerdo grupal con las cuestiones planteadas). De los 24 ítems restantes propuestos a la reconsideración de los expertos se lograron consensuar 9 más en la segunda ronda ( 8 de acuerdo grupal con el ítem planteado y 1 de desacuerdo grupal). 
Al final del proceso, el panel alcanzó consenso de opiniones en el $75 \%$ de los contenidos de la encuesta. En 15 ítems, el panel de expertos mostró un criterio indeterminado tras las dos rondas. En la tabla I se detallan los resultados globales del proyecto con sus estadísticos correspondientes, indicándose para cada ítem los estadísticos de centralización (puntuaciones de la mediana y media), el rango intercuartílico como estadístico de dispersión y el porcentaje de panelistas a favor de las propuestas.

El resultado detallado del consenso alcanzado en cada una de las áreas temáticas analizadas fue:

1. DN periférico: tratamiento tópico versus sistémico. Se consensúan 6 de los 11 ítems evaluados.

2. DN posquirúrgico, postraumático y muñones dolorosos. Se consensúan los 12 ítems evaluados.

3. Neuralgia posherpética, intercostal y del trigémino. Se consensúan 6 de los 9 ítems evaluados.

4. DN periférico por atrapamiento. Se consensuan los 8 ítems evaluados.

5. Síndrome doloroso regional complejo. Se consensúan 6 de los 11 ítems evaluados. En la figura 1 se resumen los resultados del apartado, por resultar objeto de particular controversia. La figura 2 detalla las opiniones expertas concretas del ítem 49 , por ser una recomendación cercana al límite del consenso grupal.

6. Neuropatía diabética y otras polineuropatías (por VIH, alcohol, toxicidad, etc.). Se consensúan 8 de los 10 ítems evaluados.

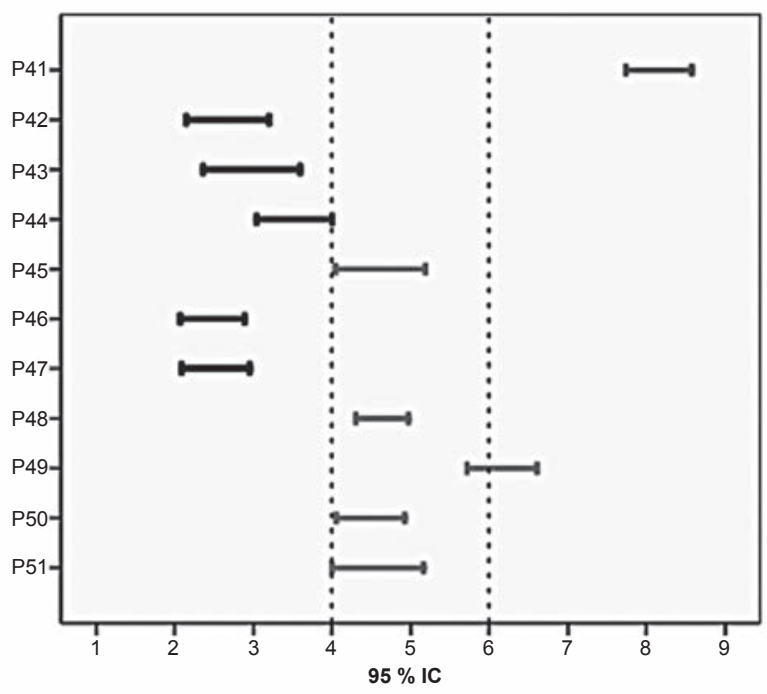

Fig. 1. Grado de consenso alcanzado por los expertos para las cuestiones planteadas sobre síndrome doloroso regional complejo. Para cada ítem representa un segmento con el recorrido del intervalo de confianza al $95 \%$ de la puntuación media del panel de expertos. El ítem 41, acuerdo por consenso grupal; los ítems 42, 43, 44, 46 y 47, rechazo por consenso grupal; los ítems 45, 48, 49, 50 y 51, criterio grupal indeterminado.

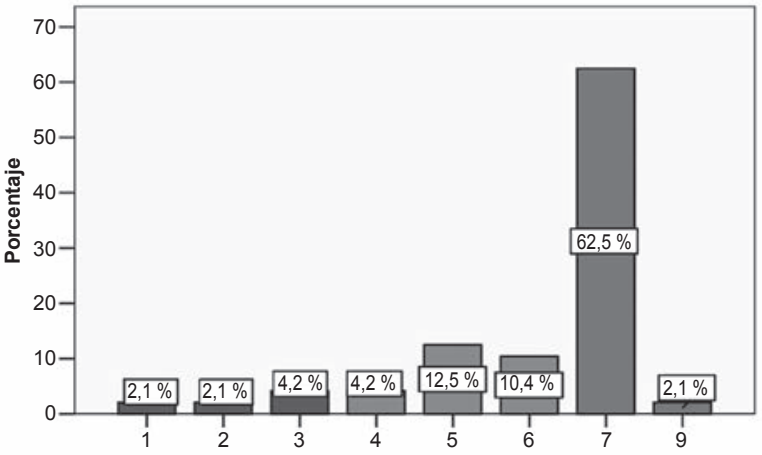

Fig. 2. Distribución de las respuestas de los expertos para el ítem 49: "La aplicación de parches de capsaicina tópica al $8 \%$ es una opción terapéutica válida en el tratamiento del dolor en el SDRC". Interpretación de opiniones: 1-3 = "desacuerdo"; 4-6 = "ni acuerdo, ni en desacuerdo"; 7-9 = "acuerdo".

\section{DISCUSIÓN}

La técnica Delphi modificada empleada en este estudio es un procedimiento de consenso profesional no presencial de amplia difusión en el contexto biomédico. Algunos de sus inconvenientes son la limitada oportunidad de realizar aclaraciones a las ideas expresadas, la necesidad de garantizar altas tasas de respuesta en los panelistas y el riesgo de influencia de sus impulsores (en la elección del panel de expertos y en la discusión de los resultados). Para controlar estos problemas, el presente trabajo ha sido codirigido por un equipo investigador multicéntrico bajo la dirección de un asesor metodológico independiente, y se han seguido procedimientos sistematizados y objetivables para la selección de panelistas (19), así como para el análisis estadístico y la interpretación de resultados (20,21).

En general, se ha obtenido un alto grado de consenso de opiniones entre los expertos consultados. En buena medida puede deberse a la gran cantidad de recomendaciones, guías clínicas y algoritmos terapéuticos disponibles sobre el DN, tanto a nivel nacional como internacional (8-13), por diferentes sociedades científicas que hacen que el manejo de este problema de salud esté muy protocolizado. La notable unanimidad sugiere, en otro sentido, que las opiniones de los panelistas probablemente reflejen el criterio general de la mayoría de expertos que atienden pacientes con DN en España, y que el panel pueda considerarse una muestra representativa de dicho colectivo profesional.

Las recomendaciones analizadas en el estudio se agruparon en seis áreas temáticas. La primera de ellas recoge aspectos generales del tratamiento del DN periférico y compara el posicionamiento clínico actual de los agentes 
tópicos frente a los tratamientos sistémicos. Sobre estos aspectos, el panel ha considerado que el uso de los fármacos tópicos como agentes de primera línea puede mejorar el cumplimiento terapéutico y la eficacia de los tratamientos, dado su favorable perfil de efectos secundarios (aunque no están exentos de ellos) y su mejor aceptación general por los pacientes. De toda la batería de criterios propuestos, recibe un particular respaldo experto la posibilidad de combinar tratamientos tópicos y sistémicos. La exploración de nuevas sinergias terapéuticas puede ser una estrategia de futuro especialmente prometedora. Sin embargo, no se alcanzó el consenso profesional en 5 de las 11 cuestiones exploradas, en particular aquellas en las que se dispone de menor evidencia y/o experiencia clínica, o sobre cuál es el grado de eficacia relativa de los fármacos tópicos frente a los sistémicos, o si todos los pacientes con DN periférico son candidatos a recibir algún tratamiento tópico. La falta de acuerdo sobre qué signos o síntomas responden mejor a los agentes tópicos puede deberse, en parte, a que se solicitó al panel la consideración conjunta de conceptos tales como la alodinia (donde mayoritariamente se acepta la utilización de la vía tópica), junto al dolor urente o las crisis lancinantes de dolor, en las cuales el papel de la vía tópica resulta más controvertido.

Las cuestiones del segundo bloque de la encuesta se refieren a un grupo de causas de DNP (dolor neuropático posquirúrgico, postraumático y muñones dolorosos) en las que la evidencia científica sobre la utilidad de la vía tópica es aún pobre. A pesar de ello, y sobre la base de la experiencia clínica conjunta de los expertos consultados, el panel alcanzó el consenso en todos los ítems. Quizá la específica afectación del sistema nervioso periférico en estos cuadros haya resultado un criterio patogénico determinante en apoyo del uso clínico de agentes tópicos que actúen selectivamente a ese nivel. No obstante, se apreció una opinión menos unánime sobre la posibilidad de hacer extensiva a estas patologías la evidencia científica existente en otros tipos de DNP, o sobre si los resultados terapéuticos demostrados solo en periodos limitados de estudio experimental permiten avalar el uso clínico generalizado de los agentes tópicos.

En el tercer bloque de encuesta (neuralgias posherpéticas, intercostal y del trigémino), los expertos lograron un claro acuerdo en considerar que el parche de capsaicina al $8 \%$ puede ser un fármaco de primera línea en el tratamiento de la neuralgia posherpética y la neuralgia intercostal localizada. Aunque una mayoría de expertos (64\%) se mostró también partidaria de la utilización del parche de capsaicina al $8 \%$ en neuralgias trigeminales $* *$ (pág. 323) del territorio V1 con respuesta insuficiente a otras alternativas terapéuticas orales (antidepresivos, antiepilépticos), y un porcentaje similar (61\%) rechazó que estos parches no puedan considerarse una opción terapéutica válida en dicho territorio, no se alcanzó la unanimi- dad suficiente requerida para el consenso estadístico. Tales opiniones, compartidas por una sustancial pero insuficiente mayoría de panelistas, son relevantes si se tiene en cuenta que expresan el apoyo experto a una indicación fuera del uso clínico actual aprobado en la ficha técnica del producto. De forma congruente, el panel estima necesario generar evidencias científicas más sólidas sobre el uso de este agente tópico en la neuralgia intercostal y trigeminal.

Todas las cuestiones planteadas en el cuarto bloque, que analiza las neuropatías por atrapamiento, fueron refrendadas por consenso de los expertos. La mayoría son referidas al síndrome del túnel carpiano, que por compresión del nervio mediano a su paso bajo el ligamento transversal del carpo produce un síndrome de dolor, parestesias, entumecimiento y debilidad en su territorio de distribución $(22,23)$. Actualmente se considera una de las causas del DN (24) de mayor incidencia e impacto sociosanitario en países desarrollados (25-27). En Estados Unidos se ha estimado que provoca un gasto cercano a los $30.000 \$$ por trabajador afectado (28). A pesar de que la causa más frecuente es la idiopática (29), se sospecha una cierta relación laboral (30-33), sin que hasta la fecha haya podido establecerse suficiente evidencia epidemiológica (34). En general, la liberación quirúrgica del nervio mediano es un procedimiento resolutivo $(35,36)$, pero algunos pacientes refieren insatisfacción con los resultados obtenidos $(37,38)$. Por otra parte existe cierta controversia sobre la necesidad de someter al paciente a un riesgo quirúrgico en casos con sintomatología leve, secundarios a otras enfermedades o con una clara relación laboral. En tal caso, las alternativas conservadoras propuestas en las guías de práctica clínica (férulas de utilización nocturna, ultrasonidos o inyecciones de esteroides) suelen lograr una respuesta parcial o temporal (22). La opinión conjunta de los expertos de este estudio parece avalar el uso tópico de agentes como el parche de capsaicina al $8 \%$ en casos leves de STC, sintomáticos pero sin signos denervativos en los estudios electroneurofisiológicos, evitando así la necesidad de titulación de agentes orales y la posible exposición a efectos secundarios sistémicos. También se considera el parche de capsaicina al $8 \%$ como una alternativa al tratamiento quirúrgico en el neuroma de Morton, en especial en aquellas situaciones donde, debido a su pequeño tamaño, su extirpación resulte difícil. Esta propuesta admite una consideración crítica, dada la escasa presencia de traumatólogos en el grupo experto (un solo especialista), por lo que el panel no dispuso de un conocimiento amplio y actualizado sobre las opciones quirúrgicas y sus resultados, lo que pudo determinar una sobrevaloración del tratamiento conservador.

El quinto bloque de la encuesta exploró diversos aspectos referidos al síndrome de dolor regional complejo (SDRC), una entidad clínica de fisiopatogenia compleja y de difícil control clínico que suscita enormes controver- 
sias a la hora de elegir el tratamiento más adecuado. En la literatura científica se proponen una gran variedad de opciones terapéuticas que abarcan desde el tratamiento médico (oral, intravenoso o transdérmico), algunas técnicas mínimamente invasivas (infiltraciones) e incluso opciones quirúrgicas radicales (como la simpatectomía). Muchos de estos tratamientos no están aún respaldados por una evidencia sólida, por lo que no es raro que en 5 de las 11 cuestiones planteadas los expertos consultados no hayan logrado consensuar sus opiniones (Fig. 1). Respecto al tratamiento por vía tópica del DN en el SDRC, la opinión mayoritaria de los expertos es no considerar esta opción como tratamiento de primera elección. Tal criterio resulta lógico ante la complejidad fisiopatogénica de este síndrome, en el que los mecanismos de sensibilización central juegan un papel determinante. Mientras que se acepta la necesidad de iniciar el tratamiento de forma precoz para mejorar el pronóstico de estos pacientes, resulta controvertida la conveniencia o no de utilizar formulaciones tópicas como coadyuvante en la fase aguda del síndrome, por la posibilidad de producir un empeoramiento del cuadro doloroso. Resulta una opinión experta compartida que los AINE en formulación tópica no consiguen un adecuado control del DN en el SDRC. En cuanto al tratamiento con parches de lidocaína o la aplicación de dimetilsulfóxido al $50 \%$, se aprecia un criterio dispar entre los expertos, que no alcanzan el consenso en su recomendación. En el primer caso, tal situación se puede justificar por la falta de estudios concluyentes sobre el anestésico local $(39,40)$, mientras que en el segundo caso, la falta de un refrendo posterior al estudio clásico que avaló su uso (41) parece explicar la escasa experiencia clínica de los panelistas consultados con dicho agente. Respecto al parche de capsaicina al $8 \%$, aunque el $65 \%$ de los expertos lo considera una opción terapéutica válida para el alivio del dolor en el SDRC (Fig. 2), en sentido estricto no se alcanza la mayoría requerida para avalar su recomendación clínica por consenso experto. El tratarse de una formulación novedosa (al $8 \%$ ) y los aún escasos datos disponibles sobre su utilidad en la literatura (42) pueden explicar la falta de unanimidad del panel. En resumen, los tratamientos por vía tópica del DN en el SDRC tienen un papel limitado, no obstante las formulaciones tópicas de capsaicina o dimetilsulfóxido podrían jugar un papel coadyuvante en pacientes seleccionados.

El último bloque de la encuesta analizó la neuropatía diabética (ND)*** (pág. 323) y otras polineuropatías. Aunque la causa más frecuente de polineuropatía en nuestro entorno es la diabetes mellitus, existen otras causas importantes asociadas a patologías como la infección $\mathrm{VIH}$, déficits nutricionales (vitamina B), enfermedades inmunológicas (sarcoidosis), vasculitis, alcoholismo, tóxicos, etc. Tan amplio espectro de causas sugiere que el tratamiento de cada paciente deba considerar no solo su sintomatología predominante, sino también la posible causa subyacente. La mayoría de los estudios disponibles se han realizado sobre la polineuropatía diabética dolorosa o la polineuropatía por VIH, por lo que las evidencias acumuladas no deberían ser directamente extrapoladas a cualquier otro tipo de polineuropatía. En estas circunstancias resultaría de utilidad tratar de sintetizar la experiencia clínica y los resultados obtenidos por los expertos en el manejo de estos cuadros mediante un consenso. Esto sería particularmente útil en el caso de los tratamientos tópicos, ya que todavía no existe ningún fármaco tópico con indicación actual en polineuropatía diabética. Capsaicina a altas dosis no está aprobada en dicha patología, aunque dispone de estudios que apuntan a este posible uso, aun sin evidencias de alto nivel. Por el contrario, es el único fármaco con eficacia demostrada en el tratamiento de la polineuropatía por VIH.

Los resultados del consenso en este bloque refrendan la seguridad del tratamiento tópico en las polineuropatías por su baja incidencia de efectos adversos y por carecer de interacciones, algo particularmente valorable en estos pacientes, con frecuencia portadores de pluripatología asociada y usuarios de múltiples tratamientos. En este sentido, la medicación tópica tendría la ventaja añadida de poder asociarse sin riesgo de interacción a cualquier otra medicación para el tratamiento del DN, logrando efectos sinérgicos al ocupar distintas dianas terapéuticas. Como contrapartida a estas ventajas, ningún tratamiento tópico tiene potencial para mejorar la comorbilidad psicológica (ansiedad, depresión y alteración del sueño) que frecuentemente presentan los pacientes con DN crónico. Quizá por ello, los expertos rechazan la propuesta de considerar los agentes tópicos como la primera opción terapéutica, salvo en casos adecuadamente individualizados según su patología subyacente y la posible comorbilidad y/o polimedicación. Por último, el panel, a la luz de la demostrada eficacia de capsaicina al $8 \%$ en la neuropatía por VIH y de la frecuente polimedicación de estos pacientes, considera la ausencia de interacciones medicamentosas una ventaja clínica especialmente relevante, hasta el punto de podría ser la primera opción terapéutica para un abordaje temprano del trastorno.

Las conclusiones del presente consenso experto se presentan en forma de recomendaciones clínicas en el Anexo 2. Estas recomendaciones para la práctica clínica deben considerarse vigentes en la fecha de elaboración de este consenso y hasta la aparición de nuevos datos científicos que justifiquen su futura revisión.

\section{AGRADECIMIENTOS}

A los 52 panelistas encuestados por su participación en el panel de expertos (ver Anexo 1). 


\section{CORRESPONDENCIA:}

Manuel Jesús Rodríguez López

Unidad del Dolor

Hospital Regional Universitario Carlos Haya

Avda. Carlos Haya, s/n

29010 Málaga

e-mail: maje1946@yahoo.es

\section{BIBLIOGRAFÍA}

1. Treede RD, Jensen TS, Campbell JN, Cruccu G, Dostrovsky JO, Griffin JW, et al. Neuropathic pain: Redefinition and a grading system for clinical and research purposes. Neurology 2008;70(18):1630-5.

2. Gálvez R, Del Pozo E, García P, Samper D, Cerón JM. Actualización en dolor neuropático. Medicina de Familia 2006;1:35-49.

3. Pérez C, Saldaña MT, Navarro A, Vilardaga I, Rejas J. Prevalence and characterization of neuropathic pain in a primary-care setting in Spain: A cross-sectional, multicentre, observational study. Clin Drug Investig 2009;29:441-50.

4. Pérez C, Ribera MV, Gálvez R, Micó JA, Barutell C, Failde I, et al. High prevalence of confirmed, but also of potential and believed, neuropathic pain in pain clinics. Eur J Pain 2013;17(3):347-56.

5. Freynhagen R, Bennett MI. Diagnosis and management of neurophatic pain. BMJ 2009;339:391-5.

6. Baron R, Binder A, Wasner G. Neuropathic pain: Diagnosis, pathophysiological mechanisms, and treatment. Lancet Neurol 2010;9(8):807-19.

7. Costigan M, Scholz J, Woolf CJ. Neuropathic pain: A maladaptive response of the nervous system to damage. Annu Rev Neurosci 2009;32:1-32.

8. Dworkin RH, O'Connor AB, Backonja M, Farrar JT, Finnerup NB, Jensen TS, et al. Pharmacologic management of neuropathic pain: Evidence-based recommendations. Pain 2007;132(3):237-51.

9. Moulin DE, Clark AJ, Gilron I, Ware MA, Watson CPN, Sessle BJ, et al. Pharmacological management of chronic neuropathic pain - Consensus statement and guidelines from the Canadian Pain Society. Pain Res Manage 2007;12(1):1321.

10. Attal N, Cruccu G, Baron R, Haanpää M, Hansson P, Jensen $\mathrm{T}$, et al. EFNS guidelines on the pharmacological treatment of neuropathic pain: 2010 revision. European Journal of Neurology 2010;17:1113-23.

11. Centre for Clinical Practice at NICE. NICE clinical guideline: The pharmacological management of neuropathic pain in adults in non-specialist settings. Issue: March 2010. National Institute for Health and Clinical Excellence. London; 2010.

12. Mailis A, Taenzer P. Evidence-based guideline for neuropathic pain interventional treatments: Spinal cord stimulation, intravenous infusions, epidural injections and nerve blocks. Pain Res Manag 2012;17(3):150-8.

13. Martínez-Salió A, Gómez de la Cámara A, Ribera MV, Montero J, Blanco E, Collado A, et al. Conferencia de Consenso: Diagnóstico y tratamiento del dolor neuropático. Med Clin (Barc) 2009;133(16):629-36.

14. Busquets C, Faulí A. Novedades en el tratamiento del dolor neuropático. Semin Fund Esp Reumatol 2012;13(03):103-9.
15. Fick DM, Cooper JW, Wade WE, Waller JL, Maclean JR, Beers MH. Updating the Beers Criteria for potentially inappropriate medication use in older adults. Results of a US Consensus Panel of Experts. Arch Intern Med 2003;163:2716-24.

16. Dalkey NC. The Delphi Method: An experimental study of group opinion. Santa Monica (California): Rand Corporation; 1969. Publication RM-59999 PR.

17. Dalkey N, Brown B, Cochran S. The Delphi Method, III: Use of Self Ratings to Improve Group Estimates. Santa Monica (California): Rand Corporation; 1969. Publication RM-6115-PR.

18. Peiró S, Portella E. El grupo nominal en el entorno sanitario. Quaderns de Salut Publica i Administració de Serveis de Salut 1. Valencia: Escola Valenciana d'Estudis per a la Salut; 2003.

19. Royle P, Waugh N. Literature searching for clinical and costeffectiveness studies used in health technology assessments reports carried out for the National Institute for Clinical Excellence appraisal system. Health Technol Assess 2003;7:1-51.

20. Goodman LA. Snowball sampling. The Annals of Mathematical Statistics 1961;32(1):148-70.

21. Holey EA, Feeley JL, Dixon J, Whittaker VJ. An exploration of the use of simple statistics to measure consensus and stability in Delphi studies. BMC Med Res Methodol 2007;7:52.

22. Ono S, Clap ham PJ, Chung KC. Optimal management of carpal tunnel syndrome. Int J Gen Med 2010;3:255-61.

23. Omer GE. Median nerve compression at the wrist. Hand Clin 1992;8(2):317-24.

24. Bouhassira D, Lanteri-Minet M, Attal N, Laurent B, Touboul C. Prevalence of chronic pain with neuropathic characteristics in the general population. Pain 2008;136(3):380-7.

25. Tanaka S, Wild DK, Seligman PJ, Behrens V, Cameron L, Putz-Anderson V. The US prevalence of self-reported tunnel carpal syndrome: 1988 National Health Interview Survey data. Am J Public Health 1994;84(11):1846-8.

26. De Krom MC, Knipschild PG, Kester AD, Thijs CT, Boekkooi PF, Spaans F. Carpal tunnel syndrome: Prevalence in the general population. J Clin Epidemiol 1992;45(4):373-6.

27. Bland JD, Rudolfer SM. Clinical surveillance of carpal tunnel syndrome in two areas of the United Kingdom. 19912001. J Neurol Neurosurg Psychiatry 2003;74(12):1674-9.

28. National Institute of Neurological Diseases and Stroke (NINDS). Available at: http://www.ninds.nih.gov/disorders/ carpal_tunnel/detail_carpal_tunnel.htm. Último acceso marzo $20 \overline{12}$.

29. De Krom MC, Knipschild PG, Kester AD, Thijs CT, Boekkooi PF, Spaans F. Carpal tunnel syndrome: Prevalence in the general population. J Clin Epidemiol 1992;45(4):373-6.

30. Atroshi I, Gummesson C, Johnsson R, Ornstein E, Ranstam J, Rosen I. Prevalence of carpal tunnel syndrome in a general population. JAMA 1999;282:153-8.

31. Brian WR, Wright AD. Spontaneous compression of both median nerves in the carpal tunnel syndrome. Lancet 1947; 1:277-82.

32. Roquelaure Y, Ha C, Nicolas G, Pélier-Cady MC, Mariot C, Descatha A, et al. Attributable risk of carpal tunnel syndrome according to industry and occupation in a general population. Arthritis Rheum 2008;59(9):1341-8.

33. Rossignol M, Stock S, Patry L, Armstrong B. Carpal tunnel syndrome: What is attributable to work? The Montreal study. Occup Environ Med 1997;54(7):519-23. 
34. Lozano-Calderón S, Anthony S, Ring D. The quality and strength of evidence for etiology: Example of carpal tunnel syndrome. J Hand Surg 2008;33A:525-38.

35. Glowacki KA, Breen CJ, Sachar K, Weiss AP. Electrodiagnostic testing and carpal tunnel release outcome. J Hand Surg Am 1996;21(1):117-21.

36. Thurston A, Lam N. Results of open carpal tunnel release: A comprehensive, retrospective study of 188 hands. Aust N Z J Surg 1997;67(5):283-8.

37. Bland JD. Do nerve conduction studies predict the outcome of carpal tunnel decompression? Muscle Nerve 2001;24(7):935-40.

38. Katz JN, Losina E, Amick BC, Fossel AH, Bessette L, Keller RB. Predictors of outcomes of carpal tunnel release. Arthritis Rheum 2001;44(5):1184-93.
39. Devers A, Galer BS. Topical lidocaine patch relieves a variety of neuropathic pain conditions: An open-label study. Clin J Pain 2000;16(3):205-8.

40. Mick G, Correa-Illanes G. Topical pain management with the $5 \%$ lidocaine medicated plaster-a review. Curr Med Res Opin 2012;28(6):937-51.

41. Zuurmond WW, Langendijk PN, Bezemer PD, Brink HE, de Lange JJ, van loenen AC. Treatment of acute reflex sympathetic dystrophy with DMSO $50 \%$ in a fatty cream. Acta Anaesthesiol Scand 1996;40:364-7.

42. Robbins WR, Staats PS, Levine J, Fields HL, Allen RW, Campbell JN, et al. Treatment of intractable pain with topical large-dose capsaicin: Preliminary report. Anesth Analg 1998;86(3):579-83. 
ANEXO I. PANEL DE EXPERTOS

\begin{tabular}{lll}
\hline Luis Aliaga Font & José María Gómez Argüelles & Consuelo Nieto Iglesias \\
María Jesús Berro Uriz & María del Carmen Gómez Martínez & María J. Pampín Conde \\
Carme Busquets Julia & José Luis Gómez Palonés & Juan Pérez Cajaraville \\
Carolina Cabanas Seijas & Jerónimo Herrera Silva & Salvadora Pérez Roilt \\
José Ignacio Calvo Sáez & Joaquín Insausti Valdivia & María Victoria Ribera Canudas \\
María Luz Cánovas Martínez & Luis Javier Jiménez Gómez & Gisela Roca Amatria \\
José Javier Carceller Ruiz & Dolores López Alarcón & María Dolores Rodrigo Royo \\
Alfonso Carregal Rañó & Ana Esther López Pérez & Fernando Rodríguez Huertas \\
Elena Catalá Puigbó & Margarita López Rouco & José Francisco Román Quiles \\
Rafael Cobos Romana & José Manuel López-Millán Infantes & Miguel Ángel Ruiz Ibán \\
Francisco Collado Collado & Antonio Martínez Salio & Juan Salmerón Cerezuela \\
Javier de Andrés Ares & Víctor Mayoral Rojals & Rogelio Sánchez de las Matas Pena \\
Carlos de Barturell Farinós & Antonio Mendoza Cerezo & María Dolores Sintes Matheu \\
Juan Carlos de la Pinta García & Jorge Montero Homs & Luis Miguel Torres Morera \\
Jesús Javier Estrada Fernández & Antonio Montes Pérez & Garbiñe Urtusagasti Orue \\
Pedro Fenollosa Vázquez & Pedro Moñino Ruiz & Domingo Ventura Vargas \\
Rafael Gálvez Mateos & José María Muñoz y Ramón & \\
Miguel Ángel García Díaz & Inmaculada Muro Castillo &
\end{tabular}

ANEXO II. PRINCIPALES RECOMENDACIONES CLÍNICAS SOBRE EL USO CLÍNICO DE LOS TRATAMIENTOS POR VÍA TÓPICA EN EL MANEJO DEL DOLOR NEUROPÁTICO PERIFÉRICO, DERIVADAS DEL CONSENSO EXPERTO

\section{Dolor neuropático periférico (DNP): tratamiento tópico versus sistémico}

- Los tratamientos tópicos, en general, resultan mejor aceptados por los pacientes, logran una mayor adherencia terapéutica y pueden ofrecer una mejor calidad de vida que los sistémicos debido a sus menores efectos adversos.

- Se recomienda introducir la vía tópica en primera línea del tratamiento del DNP y se promueve la terapia combinada, tópica y sistémica, del trastorno.

- Se recomienda el uso de fármacos con dianas farmacológicas periféricas en el tratamiento tópico del DNP.

- No se ha conseguido un criterio experto conjunto sobre la eficacia relativa de los tratamientos por vía tópica y sistémica, ni sobre la posible mejor respuesta a los agentes tópicos de los signos o síntomas positivos (por ejemplo, alodinia, disestesias, crisis lancinantes y dolor urente).

- No se ha conseguido un criterio experto conjunto sobre si todos los pacientes con DNP son candidatos a tratamiento tópico.

\section{Dolor neuropático posquirúrgico, postraumático y muñones dolorosos}

- El frecuente fracaso terapéutico en estos cuadros se debe a un diagnóstico incorrecto y a la dificultad intrínseca de su tratamiento. Se considera de gran utilidad el uso de la escala de valoración DN4.

- Los antidepresivos (tricíclicos y duales) y los ligandos alfa-2-delta de los canales de calcio se consideran como tratamientos de primera línea de estos cuadros.

- Dada la afectación del SNP en este tipo de lesiones parece útil emplear tratamientos tópicos que actúen a dicho nivel. Se recomienda facilitar el cumplimiento terapéutico con agentes tópicos que requieran un reducido número de aplicaciones.

- La combinación del parche de capsaicina al $8 \%$ con otros fármacos orales de primera línea es una alternativa eficaz para el tratamiento del DNP y permite reducir el uso de medicación oral concomitante. 
ANEXO II (CONT.). PRINCIPALES RECOMENDACIONES CLÍNICAS SOBRE EL USO CLÍNICO DE LOS TRATAMIENTOS POR VÍA TÓPICA EN EL MANEJO DEL DOLOR NEUROPÁTICO PERIFÉRICO, DERIVADAS DEL CONSENSO EXPERTO

\section{Dolor neuropático posquirúrgico, postraumático y muñones dolorosos}

- El parche de capsaicina al $8 \%$ se puede utilizar como primera opción de tratamiento en dolor neuropático posquirúrgico, postraumático y muñones dolorosos. Se recomienda el uso en fase temprana para reducir el riesgo de sensibilización central a largo plazo.

\section{Neuralgia posherpética (NPH), intercostal y del trigémino}

- El parche de capsaicina al $8 \%$ se puede considerar un fármaco de primera línea en el tratamiento de la NPH, indicación para la que puede tener más ventajas que inconvenientes.

- En un paciente con neuralgia intercostal localizada, el parche de capsaicina al $8 \%$ puede ser una opción válida de tratamiento, incluso como primera opción terapéutica.

- En caso de respuesta parcial con la aplicación del primer parche de capsaicina al $8 \%$ en casos de NPH, se puede intentar una segunda aplicación pasados dos meses.

- Aunque la mayoría de los expertos no considera el territorio trigeminal como una contraindicación al parche de capsaicina al $8 \%$, y lo valoraría como alternativa terapéutica ante una respuesta insuficiente a otros fármacos por vía oral (antiepilépticos, antidepresivos), no se alcanzó un criterio experto consensuado en la materia.

- Aunque desde un punto de vista fisiopatológico se entiende la indicación del tratamiento con parche de capsaicina al $8 \%$ en todo tipo de DNP, el panel recomienda investigar y generar más evidencias sobre este agente en el tratamiento de la neuralgia intercostal y trigeminal.

\section{Dolor neuropático periférico por atrapamiento}

- Tanto la cirugía abierta tradicional como las técnicas de mínima incisión y las técnicas endoscópicas son abordajes quirúrgicos apropiados del síndrome de túnel carpiano (STC), pero la utilización del parche de capsaicina al $8 \%$ es una alternativa posible de tratamiento conservador en estos pacientes.

- En las neuropatías por atrapamiento, los tratamientos tópicos tienen menos efectos adversos generales, no precisan titular la dosis y no presentan las interacciones farmacológicas propias de los tratamientos sistémicos.

- En el STC leve o moderado sin signos de denervación en la electromiografía estaría indicado el tratamiento conservador y, entre ellos, la vía tópica.

- En el tratamiento del STC el parche de capsaicina al $8 \%$ permite aliviar los síntomas localizados sin los efectos secundarios de los tratamientos orales. Cuanto antes se utilice menos sensibilización central y resistencias terapéuticas tendrá el paciente, siendo mayor la posibilidad de respuesta terapéutica.

- En el neuroma de Morton de pequeño tamaño, el parche de capsaicina al $8 \%$ puede aconsejarse como primera opción antes de plantear un tratamiento quirúrgico.

\section{Síndrome de dolor regional complejo (SDRC)}

- Se recomienda el inicio temprano del tratamiento en el SDRC para reducir sus posibilidades de cronificación. Los expertos no aconsejan inmovilización sistemática de la extremidad/extremidades afectadas.

- El uso de opioides por vía oral no contraindica el tratamiento concomitante con parches de opioides transdérmicos, aunque estos últimos no se consideran agentes de primera elección en el tratamiento del SDRC.

- Los agentes tópicos no se consideran el tratamiento de elección para lograr el control del SDRC, pero resulta controvertida entre los expertos la necesidad de contraindicar cualquier tipo de tratamiento tópico durante la fase aguda ("caliente") del SDRC.

- Los antiinflamatorios no esteroideos (AINE) aplicados de forma tópica no consiguen un adecuado control del dolor en el SDRC. 
ANEXO II (CONT.). PRINCIPALES RECOMENDACIONES CLÍNICAS SOBRE EL USO CLÍNICO DE LOS TRATAMIENTOS POR VÍA TÓPICA EN EL MANEJO DEL DOLOR NEUROPÁTICO PERIFÉRICO, DERIVADAS DEL CONSENSO EXPERTO

\section{Síndrome de dolor regional complejo (SDRC)}

- Sobre la base de la experiencia del panel experto, no se logra una recomendación experta consensuada sobre la posible utilidad de lidocaína tópica, ni del dimetilsulfóxido al 50 \% para aliviar el dolor en la zona de aplicación en el SDRC.

- Aunque una mayoría de expertos considera la aplicación de parches de capsaicina tópica al $8 \%$ como una opción terapéutica válida en el tratamiento del dolor en el SDRC, no resulta suficiente para considerarlo una recomendación de consenso. Los expertos tampoco recomiendan reservar este agente solo para cuando hayan fracasado otros tratamientos tópicos.

\section{Neuropatía diabética (ND) y otras polineuropatías (por VIH, alcohol, toxicidad, etc.)}

- Se recomienda una evaluación clínica precisa de la situación neurológica y un tratamiento individualizado del paciente con polineuropatía diabética para lograr éxito terapéutico.

- Aunque el tratamiento tópico puede reducir el riesgo de interacciones medicamentosas y esto puede resultar una ventaja clínica muy relevante en este tipo de pacientes, el panel experto no avala su uso como tratamiento de primera elección en las polineuropatías.

- El parche de capsaicina al $8 \%$ es el único agente que ha demostrado eficacia en la neuropatía por VIH, a través del bloqueo de los canales TRPV1, reduciendo además la incidencia de efectos adversos del tratamiento. En este tipo de pacientes el abordaje temprano con tratamientos tópicos podría ser la primera opción terapéutica.

\section{NOTAS}

**Precauciones especiales de empleo de Qutenza $^{\circledR}$ : nunca se utilizará en la cara o por encima de la línea de nacimiento del cabello, ni en la proximidad de mucosas.

***Qutenza ${ }^{\circledR}$ está indicado para el tratamiento del dolor neuropático periférico en adultos no diabéticos, solo o en combinación con otros medicamentos para el dolor. 